\title{
EFFECT OF VISCOSITY ON SLIP BOUNDARY CONDITIONS IN RAREFIED GAS FLOWS
}

\author{
Nam T. P. Le $\mathbf{e}^{1,2, *}$ \\ ${ }^{1}$ Industrial University of Ho Chi Minh City, Vietnam \\ ${ }^{2}$ Ton Duc Thang University, Ho Chi Minh City, Vietnam \\ *E-mail: letuanphuongnam@tdtu.edu.vn
}

Received: 17 January 2019 / Published online: 25 July 2019

\begin{abstract}
The viscosity of gases plays an important role in the kinetic theory of gases and in the continuum-fluid modeling of the rarefied gas flows. In this paper we investigate the effect of the gas viscosity on the surface properties as surface gas temperature and slip velocity in rarefied gas simulations. Three various viscosity models in the literature such as the Maxwell, Power Law and Sutherland models are evaluated. They are implemented into OpenFOAM to work with the solver "rhoCentralFoam" that solves the Navier-Stokes-Fourier equations. Four test cases such as the pressure driven backward facing step nanochannel, lid-driven micro-cavity, hypersonic gas flows past the sharp 2555-deg. biconic and the circular cylinder in cross-flow cases are considered for evaluating three viscosity models. The simulation results show that, whichever the first-order or second-order slip and jump conditions are adopted, the simulation results of the surface temperature and slip velocity using the Maxwell viscosity model give good agreement with DSMC data for all cases studied.
\end{abstract}

Keywords: Sutherland; Power Law; Maxwell viscosity models; rarefied gas flows; slip velocity; surface gas temperature.

\section{INTRODUCTION}

The accuracy of the Navier-Stokes-Fourier (N-S-F) simulations for rarefied and microscale gas flows depends on the slip velocity and temperature jump conditions, and also the constitutive relations supplied, such as the viscosity-temperature relation, thermal conductivity and heat capacity. We did an investigation for the slip and jump conditions in [1] to find the most suitable choice of slip velocity and temperature jump conditions for rarefied gas simulations. Flow regimes in rarefied gas dynamics are characterized by the Knudsen number, $\mathrm{Kn}$, defined as the ratio of gas mean free path (i.e. the average distance a molecule moves between successive intermolecular collisions) to a characteristic length of the vehicle body, as free molecular $(K n \geq 10)$, transition regime $(0.1 \leq K n \leq 10)$, slip regime $\left(0.001 \leq K n \leq 0.1^{3}\right)$, and continuum regime $(K n \leq 0.001)$. 
The CFD method, which solves the Navier-Stokes-Fourier (N-S-F) equations with appropriate slip and jump conditions, may simulate successfully rarefied gas flows in the slip regime, up to a Knudsen number of 0.1. The Direct Simulation Monte Carlo (DSMC) method is a commonly used to investigate the rarefied gas flows. But this method is also very expensive both in computational time and memory requirements.

The viscosity affects to the accuracy of the N-S-F simulation results through the shear stress, heat transfer and the Maxwellian mean free path presented in the slip velocity and temperature jump conditions. In gas microflows, the mean free path of the gas molecules becomes significant relative to the characteristic dimension of the microdevices. The action of viscosity can be achieved from a consideration of the transfer of molecular momentum between two contiguous layers of the mass flow. Momentum is carried by the molecules from one layer to the other both by direct translation and by intermolecular collisions. If this transfer process is undergone then viscous flow occurs [2]. So the viscosity of gases played an important role in the kinetic theory of gases and rarefied gas simulations. Various viscosity models such as the constant viscosity, Power Law and Maxwell viscosity models were investigated for one-dimensional (1D) shock structure by the CFD and DSMC methods [3,4]. The Maxwell viscosity model gave good simulation results of the shock structure in comparing with experimental data [5]. The Sutherland and Power Law viscosity models have been commonly using in CFD simulations. The viscosity of real gases can be matched by a power law over a small temperature range only, because the long-range attractive forces (the van der Waals forces) are ignored. More realistic is the Sutherland potential which combines a short-range hard sphere repulsion with a long-range inverse $6^{\text {th }}$ power attractive potential [6]. So far there is not yet any comparison between these viscosity models in two-dimensional (2D) rarefied gas simulations. In this paper three various viscosity models found in the literature such as Sutherland, Power Law and Maxwell viscosity models are numerically investigated to evaluate their performance in rarefied gas flows in the slip regime $(K n \leq 0.1)$.

Four cases such as the pressure driven backward facing step nanochannel [7], lid driven micro-cavity, [8], hypersonic gas flow past the sharp 25-55-deg. biconic [9] and a circular cylinder in cross-flows [10] are considered to investigate the effects of viscosity on the slip velocity and surface gas temperature. The first-order and second-order slip conditions in [11-13] are adopted to simulate four cases within the OpenFOAM framework [14]. The simulation results of the surface gas temperature and slip velocity are compared with the DSMC data published in [11-14] to find out which viscosity model should be used for predicting the surface quantities in rarefied gas flow simulations.

\section{VISCOSITY MODELS}

In 2D simulations, the Maxwell viscosity model employed for 1D simulation in [3], $\mu=2 \sqrt{m k_{B} T / \pi} / 3 \pi d^{2}$, is slightly corrected that would be presented below; where $m$ is mass of a molecule; $k_{B}$ is the Boltzmann constant, $d$ is the molecular diameter and $T$ is temperature. Whichever model for viscosity, $\mu$, is adopted, the coefficient of thermal conductivity, $k$, may be determined from the formula $k=c_{p} \mu / \operatorname{Pr}$ where the Prandtl number, Pr, is assumed to be constant and $c_{p}$ is the constant pressure specific heat. 
When two molecules collide with each other, energy, momentum and mass are all conserved. If we examine the transport of momentum it means we have been studying viscosity of a gas [15]. The phenomena of viscosity occur in a gas when it undergoes a shearing motion. It is found experimentally that the stress acts in the gas across any plane perpendicular to the direction of the velocity gradient is not only the nature of a simple pressure normal to the plane but also contains a tangential or shearing component. The net transfer of momentum of molecules crossing the plane appears as the effect of viscosity for a two-dimensional gas and is computed by [15]

$$
\mu=\sqrt{\frac{m k_{B}}{\pi}} \frac{1}{\pi d^{2}} T^{0.5}
$$

This equation of gas viscosity was inspired by Maxwell, so-called the Maxwell viscosity model. In comparison with the Maxwell viscosity model mentioned-above in 1D simulation, the factor (2/3) vanishes in the 2D Maxwell viscosity model. Observing that according to the kinetic theory of gases, $\mu$ is proportional to $T^{0.5}$, and molecular diameter.

In the other approach, the viscosity also depends on the intermolecular force that determines how molecules interact in collision with each other. The Power Law viscosity model is simple and expressed in the well-known relation,

$$
\mu=A_{P} T^{s}, \quad \text { where } \quad s=\frac{1}{2}+\frac{2}{v-1},
$$

where $A_{P}$ is a constant of proportionality and depends on the reference temperature. The accuracy of the Power Law model depends on the exponent $s$ over the range of temperature. The values $v$ and $s$ for the intermolecular force law can be determined from the limiting theoretical cases $[15,16]$. The values $s$ and $v$ for the intermolecular force law for hard-sphere molecules are $v=\infty, s=0.5$, and $v=5, s=1$ corresponding to Maxwellian molecules. Real molecules generally have $v$ ranging from 5 to 15 [15]. Moreover, the values $s$ is suitably chosen to satisfy experimental data [5]. However, the viscosity can match by a power law over a small temperature range only, because the attractive forces are ignored. It is seen that the Maxwell viscosity model above (Eq. (1)) can be re-written in the Power Law form $\mu=A_{M} T^{s}$, with $A_{M}=\left(\sqrt{m k_{B} / \pi}\right) / \pi d^{2}$ and $s=0.5$.

The Sutherland viscosity model is more complicated than Power Law viscosity model. It adds a weak attractive force to the intermolecular force which is more realistic. This law is valid only if the attractive force of the intermolecular force is small. The Sutherland model is expressed as.

$$
\mu=A_{s} \frac{T^{1.5}}{T+T_{s}}
$$

where $A_{S}$ and $T_{S}$ are constant. The coefficient $A_{S}$ depends on the reference temperature, and $T_{S}$ is a measure of strength of the attractive force [6]. These constants are interpreted from experimental data and taken in $[3,5,6]$ to fit the viscosity as accurate as possible. The values $A_{S}$ and $T_{S}$ for different gases in the range of gas temperature from 58 to $1000 \mathrm{~K}$ are given in $[3,17]$ : for argon $A_{S}=1.93 \times 10^{-6}\left(\mathrm{~Pa} . \mathrm{S} / \mathrm{K}^{-1 / 2}\right)$ and $T_{S}=142 \mathrm{~K}$, and for nitrogen 
$A_{S}=1.41 \times 10^{-6}\left(\right.$ Pa.s $\left./ \mathrm{K}^{-1 / 2}\right)$ and $T_{S}=111 \mathrm{~K}$. Finally, the macroscopic viscosity model using for DSMC simulations [10] is expressed as follows,

$$
\mu=\mu_{\text {ref }}\left(\frac{T}{T_{\text {ref }}}\right)^{\omega} \text {, where } \mu_{\text {ref }}=\frac{15 \sqrt{\pi m k_{B} T_{\text {ref }}}}{2 \pi d_{\text {ref }}^{2}(5-2 \omega)(7-2 \omega)},
$$

where $\omega$ is the variable-hard-sphere temperature exponent. This model requires a reference temperature, $T_{\text {ref }}$, reference diameter, $d_{\text {ref }}$ and the exponent, $\omega$. Eq. (4) can be written in the power-law form $\mu=A T^{s}$ if we set the constant $A=\mu_{\text {ref }} / T_{\text {ref }}^{-\omega}$ and $s=\omega$.

The open source CFD software, OpenFOAM [11], is used in the present work. It uses finite volume numeric to solve systems of partial differential equations ascribed on any 3-dimensional unstructured mesh of polygonal cells. The Maxwell viscosity model presented in the form of $\mu=A_{M} T^{s}$, the Power Law and the Sutherland viscosity models are implemented into OpenFOAM to work with the CFD solver "rhoCentralFoam" that solves the N-S-F equations.

\section{SLIP VELOCITY AND TEMPERATURE JUMP CONDITIONS}

In this paper, we focus on the numerical evaluation of viscosity models in rarefied gas flows in slip regime $(K n \leq 0.1)$. So the simple slip and jump conditions are selected in the present work. The first-order conventional Maxwell slip boundary condition can be expressed in vector form as [11]

$$
\mathbf{u}+\left(\frac{2-\sigma_{u}}{\sigma_{u}}\right) \lambda \nabla_{n}(\mathbf{S} \cdot \mathbf{u})=\mathbf{u}_{w}-\left(\frac{2-\sigma_{u}}{\sigma_{u}}\right) \frac{\lambda}{\mu} \mathbf{S} \cdot\left(\mathbf{n} \cdot \boldsymbol{\Pi}_{m c}\right)-\frac{3}{4} \frac{\mu}{\rho} \frac{\mathbf{S} \cdot \nabla T}{T},
$$

where $\boldsymbol{\Pi}_{m c}=\mu\left((\nabla \mathbf{u})^{T}-\left(\frac{2}{3}\right) \mathbf{I} \operatorname{tr}(\nabla \mathbf{u})\right)$. The right hand side of Eq. (5) contains 3 terms that are associated with (in order): the surface velocity, the so-called curvature effect, and thermal creep; $p$ is the gas pressure; $\mathbf{u}$ and $\mathbf{u}_{w}$ is the velocity and the wall velocity, respectively; $\mathbf{n}$ is the unit outward normal vector; $\mathbf{S}=\mathbf{I}-\mathbf{n n}$ where $\mathbf{I}$ is the identity tensor, removes normal components of any non-scalar field; $T$ is the transpose and $t r$ is the trace. The tangential momentum accommodation coefficient, $\left(0 \leq \sigma_{u} \leq 1\right)$, determines the proportion of molecules reflected from the surface specularly (equal to $1-\sigma_{u}$ ) or diffusely (equal to $\sigma_{u}$ ). The Maxwellian mean free path is calculated by [15]

$$
\lambda=\frac{\mu}{\rho} \sqrt{\frac{\pi}{2 R T}} .
$$

Experimental observations show that the temperature of a rarefied gas at a surface is not equal to the wall temperature, $T_{w}$. This difference is called the "temperature jump" and is driven by the heat flux normal to the surface. The Smoluchowski boundary condition can be written [12]

$$
T+\frac{2-\sigma_{T}}{\sigma_{T}} \frac{2 \gamma}{(\gamma+1) \operatorname{Pr}} \lambda \nabla_{n} T=T_{w}
$$

where $\gamma$ is the specific heat ratio; $\sigma_{T}$ is thermal accommodation coefficient $\left(0 \leq \sigma_{T} \leq 1\right)$. Perfect energy exchange between the gas and the solid surface corresponds to $\sigma_{T}=1$, 
and no energy exchange to $\sigma_{T}=0$. The second order velocity slip boundary condition for a planar surface can be expressed as follows [13]

$$
\mathbf{u}=-A_{1} \lambda \nabla_{n}(\mathbf{S} \cdot \mathbf{u})-A_{2} \lambda^{2} \nabla_{n}^{2}(\mathbf{S} \cdot \mathbf{u})+\mathbf{u}_{w},
$$

where $A_{1}$ and $A_{2}$ are the first and second order coefficients. It was assumed there is no more heat flux along the surface. The values $A_{1}$ and $A_{2}$ are proposed either from theory or from experiment. Recently we suggested the second order jump condition in a new form as follows [13]

$$
T=-\frac{2 \gamma}{\gamma+1} \frac{1}{\operatorname{Pr}}\left(C_{1} \lambda \nabla_{n} T+C_{2} \lambda^{2} \nabla_{n}^{2} T\right)+T_{w}
$$

where $C_{1}$ and $C_{2}$ are the first and second order coefficients.

The first-order and the second-order slip and jump conditions were also implemented into OpenFOAM presented in our previous work $[1,14,17]$ to employ with the solver "rhoCentralFoam" for running all CFD simulations. In this solver, the laminar N-S-F equations are numerically solved using a finite volume discretization and high-resolution central schemes to simulate high-speed viscous flows, and a calorically perfect gas for which $p=\rho R T$ is assumed.

\section{NUMERICAL RESULTS AND DISCUSSIONS}

Four cases such as the pressure driven backward facing step nanochannel, $K n=$ 0.025 [7], lid driven micro-cavity, $K n=0.05$ [8], hypersonic gas flows past the sharp 25-55deg. biconic with Mach number $M a=15.6$ [9], and past a circular cylinder in cross-flow, $M a=10, K n=0.01$ [10] are considered in the present work. The characterized lengths to calculate the $K n$ numbers for cases are 1) the height of the channel, $H, 2$ ) the length of cavity, $L, 3)$ diameter of the biconic base, $2 R$, and 4 ) the diameter of cylinder, $D$. Their values are found in Tab. 1. In all CFD simulations at the walls, the slip and jump boundary conditions are applied for $(T, \mathbf{u})$, and zero normal gradient condition is set for $p$. For the step nanochannel case, $p_{\text {in }}$ and $T_{\text {in }}$ are set at the entrance, and $p_{\text {out }}$ is set at the outlet. The gas flow is driven by the pressure gradient, and the velocity of gas flow depends on the pressure gradient. The velocity is then calculated explicitly, and the Neumann type is used for both inlet and outlet for velocity. Zero normal gradient condition is applied for $\mathbf{u}$ at the entrance and exit, and for $T$ at the exit of channel, seen in Fig. 1(a). For the lid-driven micro-cavity case, initial pressure and temperature are set as initial values in the computational domain, shown in Fig. 1(b). For the two-dimensional axisymmetric biconic

Table 1. Gas properties and characterized lengths of all cases

\begin{tabular}{ccccccc}
\hline Cases & $\omega$ & $T_{\text {ref }}(\mathrm{K})$ & $d_{\text {ref }}(\mathrm{m})$ & $m(\mathrm{~kg})$ & Gas & Characterized lengths \\
\hline Step nanochannel & 0.74 & 273 & $4.17 \times 10^{-10}$ & $46.5 \times 10^{-27}$ & Nitrogen & $H=17.09 \mathrm{~nm}$ \\
Micro-cavity & 0.81 & 273 & $4.17 \times 10^{-10}$ & $66.3 \times 10^{-27}$ & Argon & $L=1 \mu \mathrm{m}$ \\
Biconic & 0.74 & 273 & $4.17 \times 10^{-10}$ & $46.5 \times 10^{-27}$ & Nitrogen & $2 R=261.8 \mathrm{~mm}$ \\
Cylinder & 0.734 & 1000 & $3.595 \times 10^{-10}$ & $66.3 \times 10^{-27}$ & Argon & $D=304.8 \mathrm{~mm}$ \\
\hline
\end{tabular}


case, the geometry is specified as a wedge of one cell thickness running along the plane of geometry. The axisymmetric wedge planes must be specified as separated patches of type "wedge", seen in Fig. 1(c). For the sharp 25-55-deg. biconic and cross-flow cylinder cases, at the inflow boundary, the freestream $(p, T, \mathbf{u})$ conditions were maintained throughout the computational process. At outflow boundary for these both cases, zero normal gradient condition are applied for $(p, T, \mathbf{u})$. At the bottom boundary of the biconic and cylinder, a symmetry boundary condition is applied to all flow variables, shown in Figs. 1(c) and 1(d).

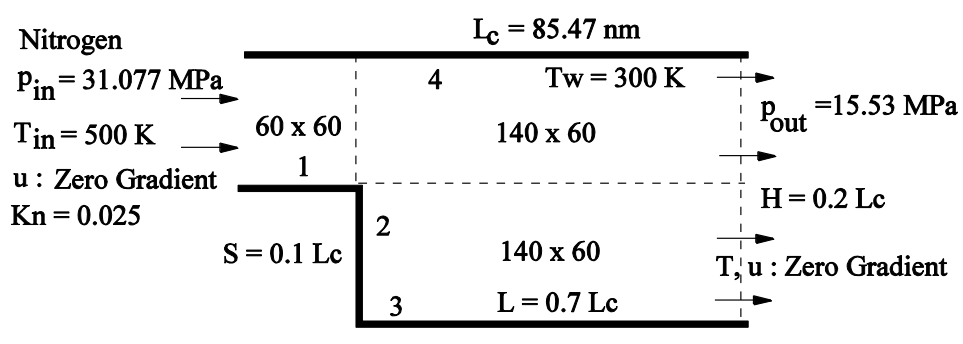

(a) Backward facing step nanochannel

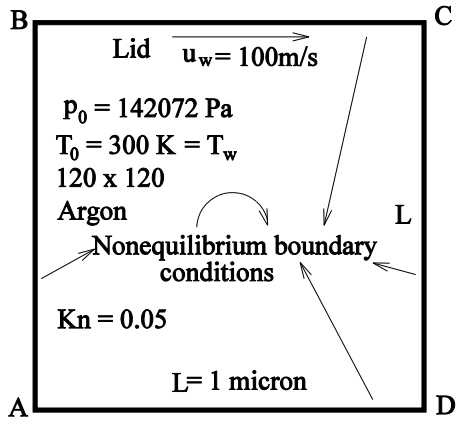

(b) Lid-driven micro-cavity

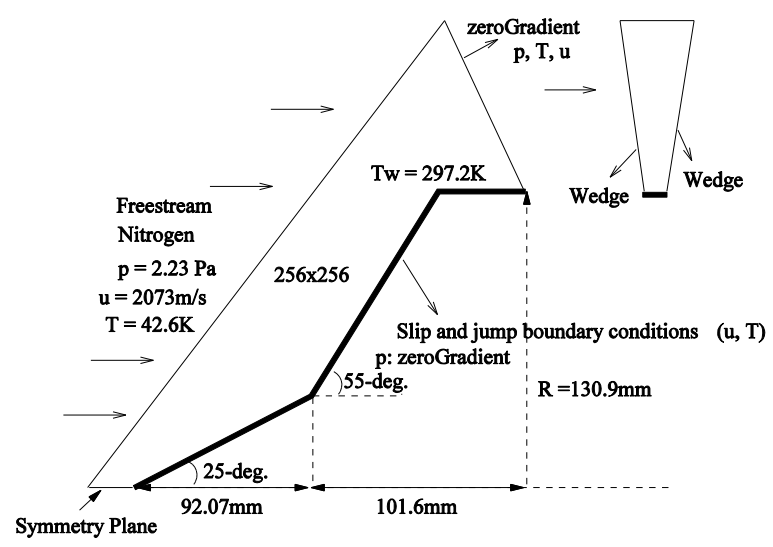

(c) Sharp 25-55-deg. biconic

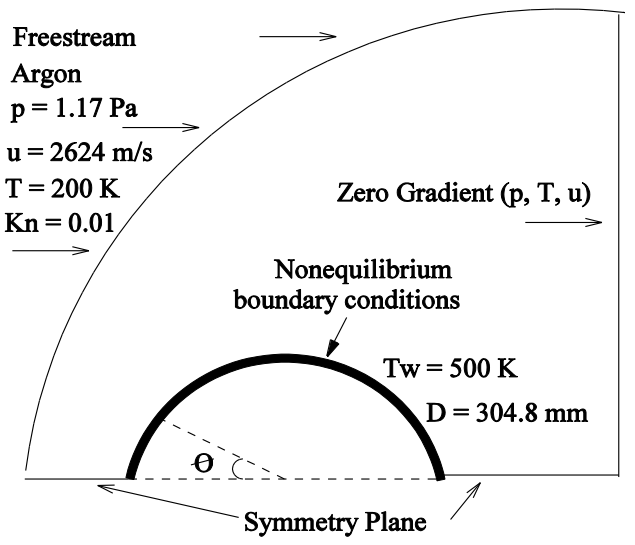

(d) Circular cylinder

Fig. 1. Numerical setups, input parameters and geometry dimensions of four cases

The geometry dimensions, numbers of cells for blocks in computational domain, input parameters and working gases of all cases are given in Fig. 1. Numbers of cells are $60 \times 60,140 \times 60$ and $140 \times 60$ for blocks of the backward facing step nanochannel case, seen in Fig. 1(a). Those are $120 \times 120$ for the cavity case, and $256 \times 256$ for the biconic case (i.e. 256 cells in the axial, streamwise direction and 256 cells in the radial, surface normal direction). For the circular cylinder case, the computational structured mesh is 
constructed to wrap around the leading bow shock with the smallest cell sizes grading near the surface $\Delta x=0.1 \mathrm{~mm}, \Delta y=1.196 \mathrm{~mm}$.

The second-order slip and jump conditions obtained good results for simulating rarefied gas microflows. So they are adopted for simulating two nano/micro-flow cases in the present work with the coefficient values $A_{1}=C_{1}=1.3$ and $A_{2}=C_{2}=0.23$ proposed in our previous work [13]. The first-order Maxwell/Smoluchowski conditions are selected for simulating hypersonic cases with the coefficients $\sigma_{T}=\sigma_{u}=1$. In the present work the CFD results would be compared with DSMC data using the values $\sigma_{T}=\sigma_{u}=1$. For a fair comparison, the viscosity should be treated as equivalent as possible between the DSMC and CFD simulations. This means the parameters $\left(m, \omega, d_{\text {ref }}, T_{\text {ref }}\right)$, that are chosen to calculate the constant $A=\mu_{\text {ref }} / T_{\text {ref }}^{-\omega}$ of the DSMC macroscopic viscosity, will be adopted for viscosity models in CFD as 1) $s=\omega$ for the Power Law viscosity model, and 2) the constant $A_{M}=\left(\sqrt{m k_{B} / \pi}\right) / \pi d_{\text {ref }}^{2}$ for the Maxwell viscosity model. These parameters of gas properties are shown and characterized lengths in Tab. 1.

\subsection{Pressure driven backward facing step nanochannel case}

In the pressure driven backward facing step nanochannel, $K n=0.025$ [7], we present the simulation results on the wall-3 of the step channel only in the streamwise direction because the separation zone is located over this wall. The surface gas temperatures increase to the peak temperature and then gradually decrease along the wall-3, seen in Fig. 2. The prediction of the Maxwell viscosity model for the gas surface temperature gives good agreement with the DSMC data [7] while the CFD other results do not. Slip velocities on the wall-3 consist of negative and positive components shown in Fig. 3. Negative ones represent the separation zone, and the distance, where indicates the negative slip velocities, is defined as the length of the separation zone. It is seen that the prediction using the Maxwell viscosity model gives better slip velocity than the CFD other results in comparing with DSMC data [7].

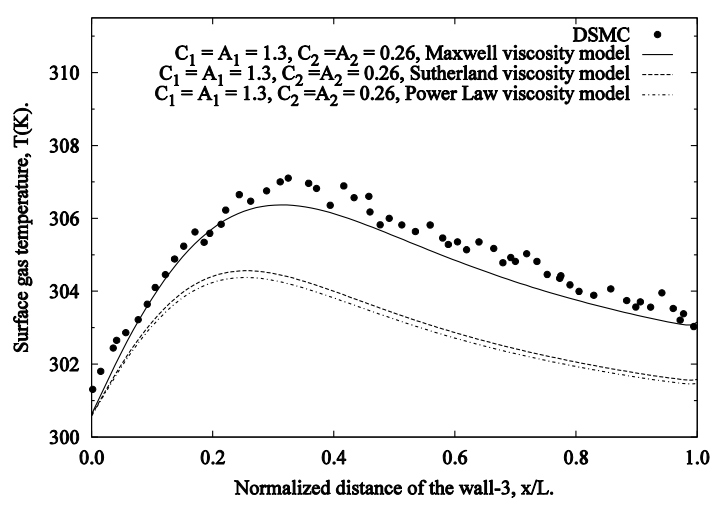

Fig. 2. Surface gas temperature along the wall$3, K n=0.025$ [7]

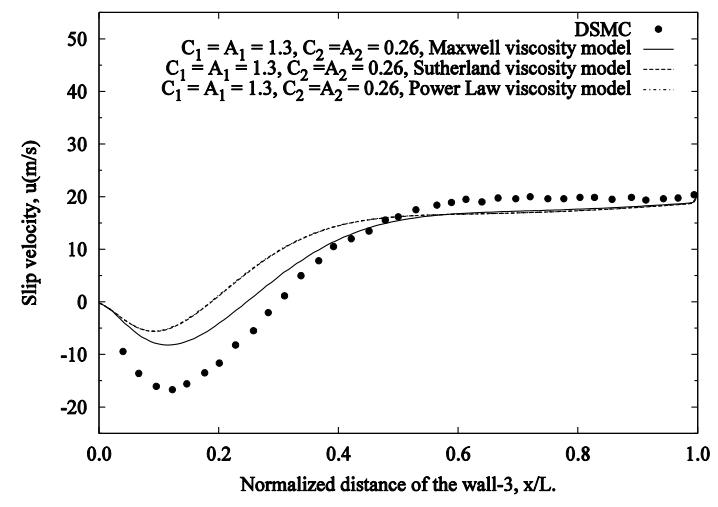

Fig. 3. Slip velocity along the wall-3, $K n=0.025$ [7] 


\subsection{Lid driven micro-cavity case}

For the lid driven micro-cavity case, $K n=0.05$ [8], the gas flow expands at the location $x / L=0$ as it is driven by the moving lid, and it is compressed at the location $x / L=1$. Considering the surface gas temperature along the lid wall, the Power Law and the Sutherland viscosity models underpredicts the temperature in the range $x / L<0.1$ in comparing with DSMC data [8] and that with the Maxwell viscosity model, seen in Fig. 4. The simulation result obtained with the Maxwell viscosity model give good agreement with DSMC data along the lid surface. At the location $x / L=1$ the gas flow is reattachment, and all simulation results show that the temperature increasing with $T>T_{w}=300 \mathrm{~K}$. It means there is viscous heat generation which results in the heat transfer from the gas to the wall toward the location $x / L=1$ of the cavity case.

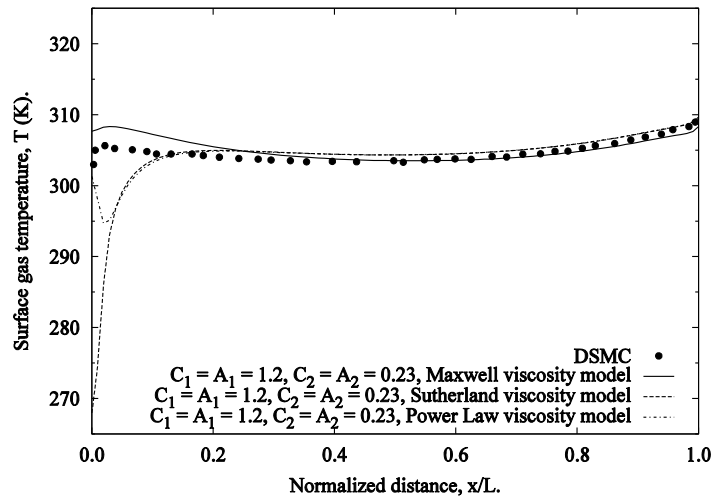

Fig. 4. Surface gas temperature along the lid wall, $K n=0.05[8]$

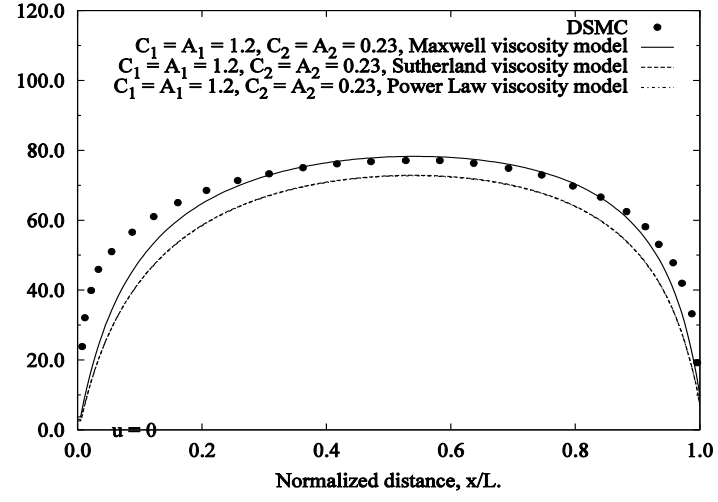

Fig. 5. Slip velocity along the lid wall, $K n=0.05[8]$

For the slip velocity along the lid wall in Fig. 5, all simulations showed that the slip velocities are very slow at the locations $x / L=0$ and $x / L=1$, and obtained the peak value around the location $x / L=0.5$. The Power Law and the Sutherland viscosity models underpredict the slip velocities along the lid surface in comparing DSMC data [8]. The simulation result using the Maxwell viscosity model is close to DSMC data while those of the Power Law and Sutherland viscosity models are not.

\subsection{Sharp 25-55-deg. biconic case}

An oblique shock forms from the tip of the first cone and locates along towards near the end of this cone, and then separates creating a shock. Latter one interacts with the oblique shock and meets the detached bow shock being formed over the second cone. A low speed recirculation zone forms at the junction between the first and the second cones in the range $0.0754 \mathrm{~m} \leq x \leq 0.1021 \mathrm{~m}$ where presents the negative slip velocity, seen in Fig. 6.

Fig. 7 compares the CFD surface gas temperatures with those of DSMC data [9]. The surface gas temperature with the Maxwell viscosity model is close to the DSMC data [9] near the tip of biconic. The surface gas temperatures obtain the peak values at the biconic tip, and thereafter rapidly decrease in the range $x \leq 0.754 \mathrm{~m}$. In this range the 
surface gas temperature predicted by the Maxwell viscosity model give good agreement with the DSMC data. There is a drop of temperature in the recirculation zone. All CFD temperatures and DSMC data are close together in $0.0754 \mathrm{~m} \leq x \leq 0.02 \mathrm{~m}$.

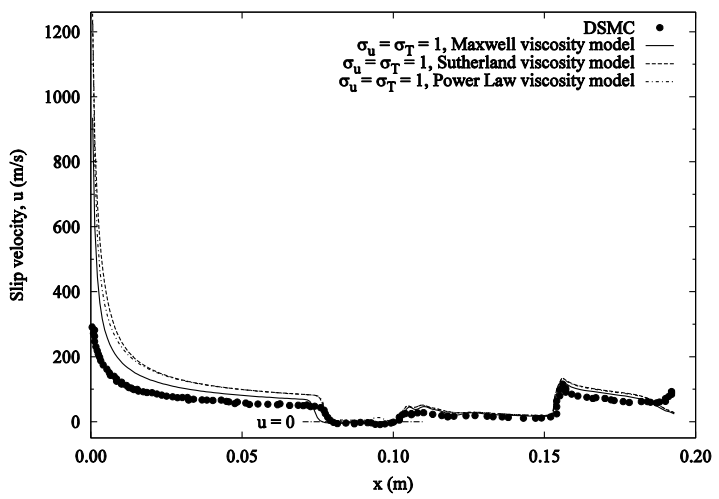

Fig. 6. Slip velocity distribution over the biconic surface [9]

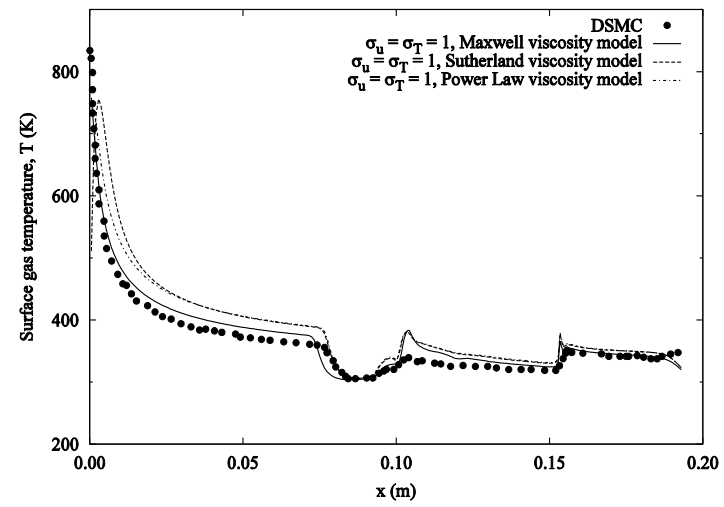

Fig. 7. Surface gas temperature distribution over the biconic surface [9]

Fig. 6 compares the CFD and DSMC [9] slip velocities along the biconic surface. Slip velocities on the biconic surface consist of negative and positive components. Negative ones represent the recirculation zone, and the distance, where indicates the negative slip velocities, is defined as the length of the recirculation zone. The slip velocities obtain the peak values at the biconic tip and then quickly decrease along the forecone until the locations $x=0.075 \mathrm{~m}$. The CFD results using the Maxwell viscosity model are close to the DSMC data. Past this zone the slip velocities increase and oscillate along the second 55 -deg. cone, and there is good agreement between all CFD results and the DSMC data in the range $0.105 \mathrm{~m} \leq x \leq 0.02 \mathrm{~m}$. Overall, the Maxwell viscosity model predicts better slip velocity than the Sutherland and the Power Law models in comparing with DSMC data.

\subsection{Cross-flow circular cylinder cases}

In the cylinder cases, various values of accommodation coefficients $\sigma_{u}=\sigma_{T}=1$, $\sigma_{u}=\sigma_{T}=0.8, \sigma_{u}=\sigma_{T}=0.6$ and $\sigma_{u}=\sigma_{T}=0.4$ are conducted for all simulations. The solver "dsmcFoam" is used to run the DSMC simulations, and generates the DSMC data. The surface gas temperatures and slip velocities are plotted against with the cylinder angle. All CFD simulations predict a higher slip velocity than the DSMC data, as seen in Figs. 8-11 for the cases $\sigma_{u}=\sigma_{T}=1, \sigma_{u}=\sigma_{T}=0.8, \sigma_{u}=\sigma_{T}=0.6$ and $\sigma_{u}=\sigma_{T}=0.4$, respectively. The DSMC and CFD slip velocities increase gradually from $0 \leq \theta \leq 13$-deg., reaching peak normalized values around the location $\theta=13-\mathrm{deg}$., and then gradually decrease in 13-deg. $\leq \theta \leq 180$-deg. The slip velocity using the Maxwell viscosity model obtains the lowest values, and are relatively close to the DSMC data. Considering the surface gas temperature, all the CFD and DSMC results are shown in Figs. 12-15 for the cases $\sigma_{u}=\sigma_{T}=1, \sigma_{u}=\sigma_{T}=0.8, \sigma_{u}=\sigma_{T}=0.6$ and $\sigma_{u}=\sigma_{T}=0.4$, respectively, in which the one using the Maxwell viscosity model is close to the DSMC data. There are differences between the CFD and DSMC temperatures along the cylinder surface. These differences 
may be explained by the calculation of the translational surface gas temperature in DSMC depending on the components of gas velocity and the slip velocity only. While that in CFD is calculated by the normal gradient of gas temperature, and is independent of the gas velocity. This leads to the profile of the DSMC temperature being very similar to that of the DSMC slip velocity.

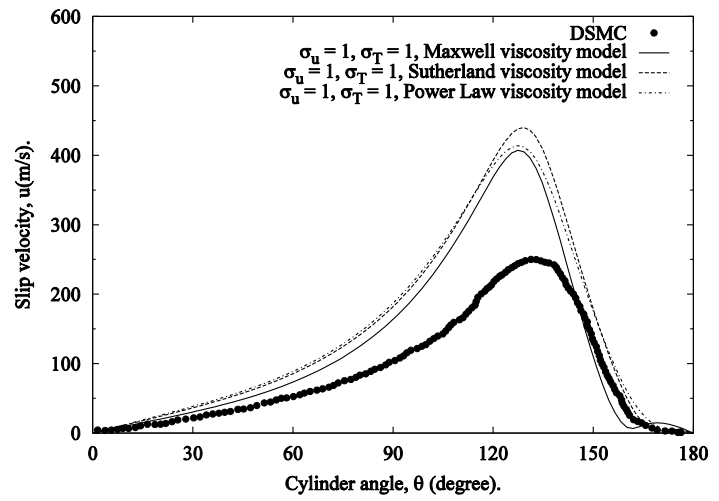

Fig. 8. Slip velocity distribution around the cylinder surface, $\sigma_{u}=\sigma_{T}=1$

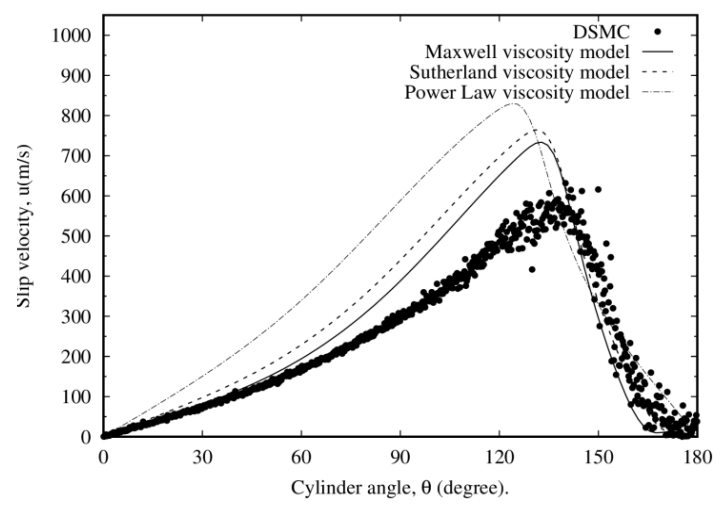

Fig. 10. Slip velocity distribution around the cylinder surface, $\sigma_{u}=\sigma_{T}=0.6$

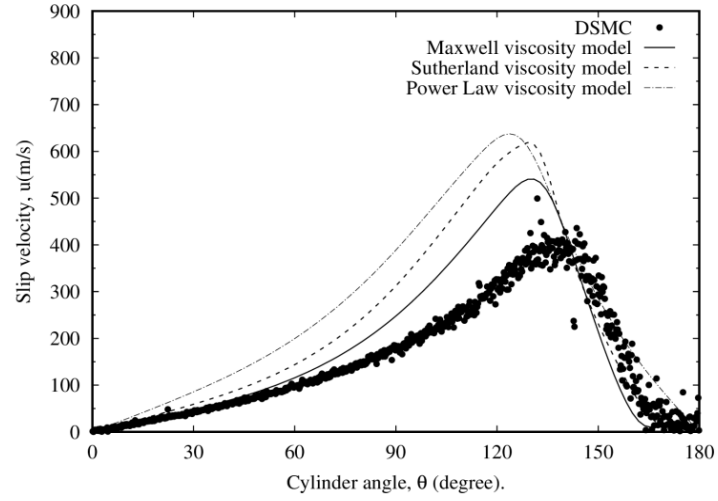

Fig. 9. Slip velocity distribution around the cylinder surface, $\sigma_{u}=\sigma_{T}=0.8$

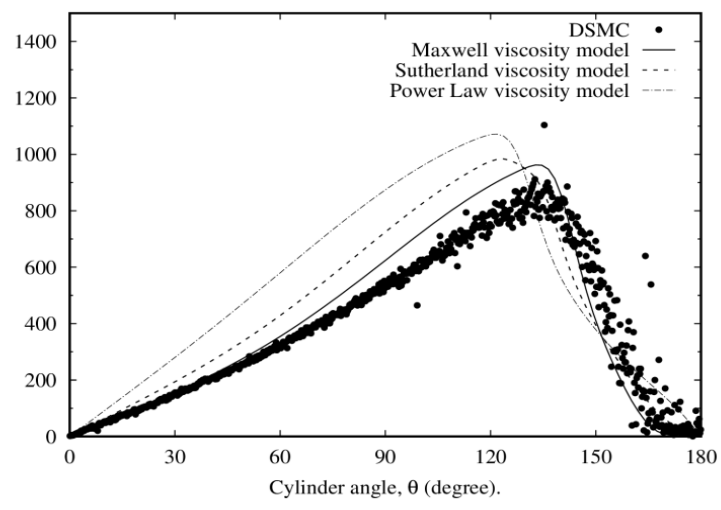

Fig. 11. Slip velocity distribution around the cylinder surface, $\sigma_{u}=\sigma_{T}=0.4$

Finally, the average errors between all CFD and DSMC simulations are shown in Tab. 2. The CFD simulations using the Maxwell viscosity model obtain the smallest average errors in comparing with those of the CFD simulations with the Power Law and Sutherland viscosity models. The reduction of thermal accommodation coefficient affects the factor $\left(2-\sigma_{T}\right) / \sigma_{T}$ in the jump temperature condition that results in the increases of the surface gas temperatures. It is also seen that the reduction of the surface accommodation effectively decreases the effect of viscosity on the flow field, and leads to the increases of the slip velocity. 


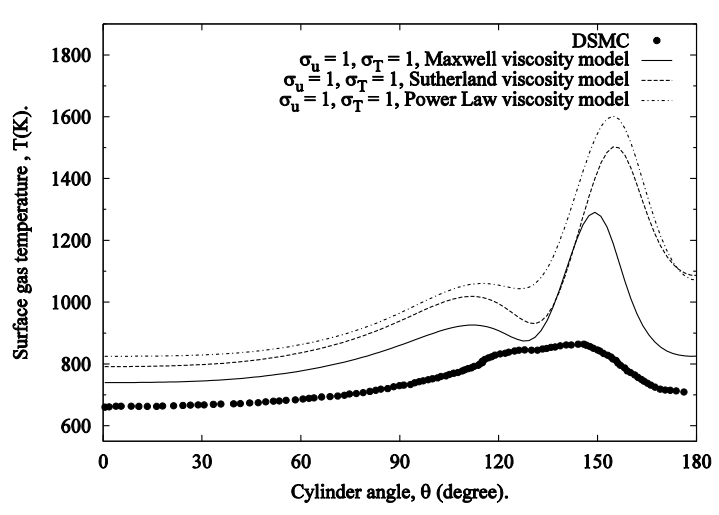

Fig. 12. Temperature jump distribution around the cylinder surface, $\sigma_{u}=\sigma_{T}=1$

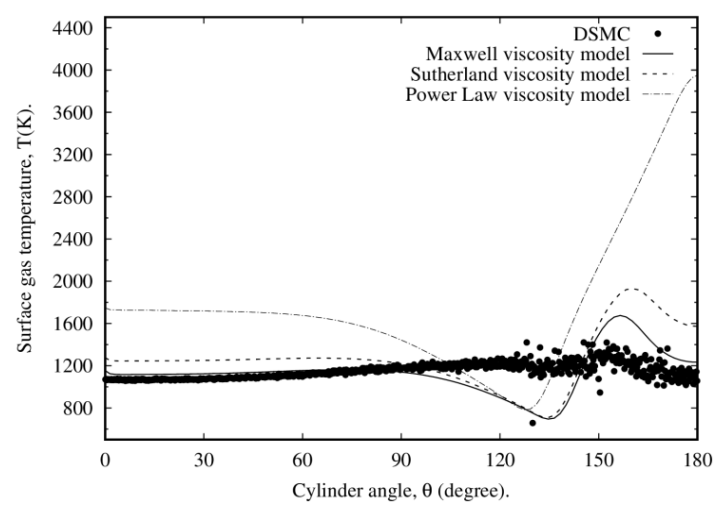

Fig. 14. Temperature jump distribution around the forebody cylinder surface,

$$
\sigma_{u}=\sigma_{T}=0.6
$$

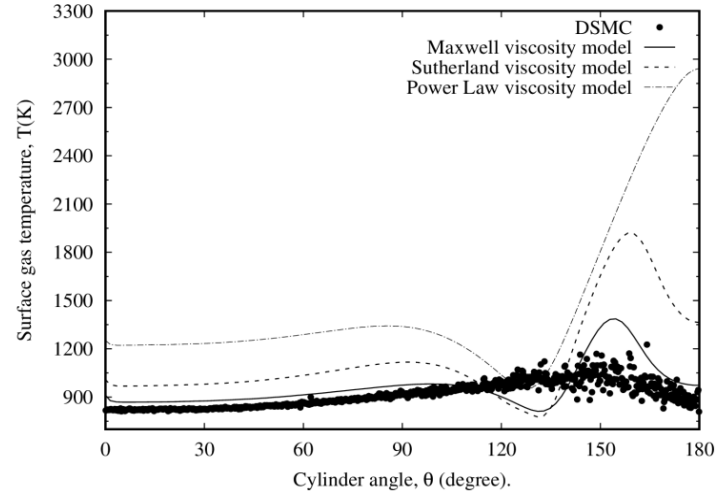

Fig. 13. Temperature jump distribution around the cylinder surface, $\sigma_{u}=\sigma_{T}=0.8$

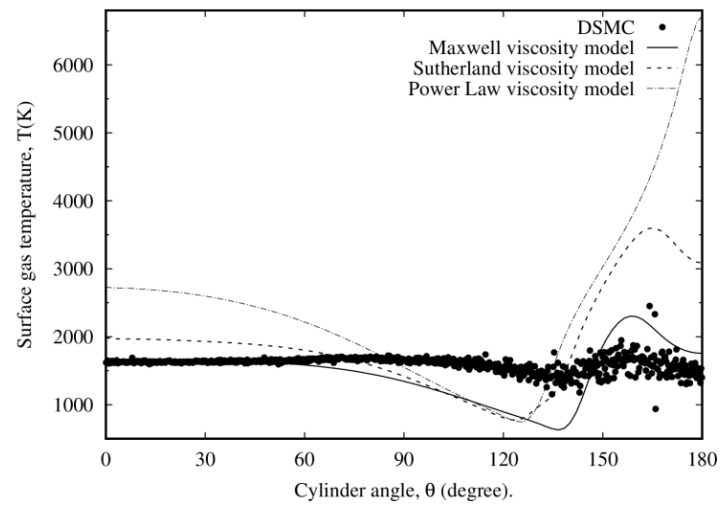

Fig. 15. Temperature jump distribution around the forebody cylinder surface,

$$
\sigma_{u}=\sigma_{T}=0.4
$$

Table 2. Average errors between the CFD and DSMC simulations of the cylinder cases

\begin{tabular}{crrrrrr}
\hline \multirow{2}{*}{ Cases } & \multicolumn{2}{c}{$\begin{array}{c}\text { Maxwell } \\
\text { viscosity model }\end{array}$} & \multicolumn{2}{c}{$\begin{array}{c}\text { Sutherland } \\
\text { viscosity model }\end{array}$} & \multicolumn{2}{c}{$\begin{array}{c}\text { Power Law } \\
\text { viscosity model }\end{array}$} \\
\cline { 2 - 7 } & \multicolumn{1}{c}{$T$} & \multicolumn{1}{c}{$\mathbf{u}$} & \multicolumn{1}{c}{$T$} & $\mathbf{u}$ & $T$ & $\mathbf{u}$ \\
\hline$\sigma_{u}=\sigma_{T}=1$ & $15.12 \%$ & $16.84 \%$ & $28.98 \%$ & $34.01 \%$ & $35.65 \%$ & $33.81 \%$ \\
$\sigma_{u}=\sigma_{T}=0.8$ & $2.84 \%$ & $13.56 \%$ & $20.90 \%$ & $34.82 \%$ & $55.34 \%$ & $61.81 \%$ \\
$\sigma_{u}=\sigma_{T}=0.6$ & $1.15 \%$ & $16.44 \%$ & $9.56 \%$ & $29.50 \%$ & $53.39 \%$ & $58.78 \%$ \\
$\sigma_{u}=\sigma_{T}=0.4$ & $8.87 \%$ & $6.70 \%$ & $16.43 \%$ & $17.55 \%$ & $49.36 \%$ & $36.53 \%$ \\
\hline
\end{tabular}




\subsection{Discussion}

Although the Sutherland viscosity model has been currently using mostly in the CFD rarefied gas simulations but the simulation results show that the Maxwell viscosity model give the good agreement with DSMC data for both the first-order and second-order slip velocity and temperature jump conditions, and with various accommodation coefficients in all cases considered. This may be explained that the Maxwell viscosity model was derived based on the net transfer of momentum since the gas molecules across any plane perpendicular in direction of velocity gradient resulting in the fixed coefficient $s=0.5$, and did not depend on the reference temperature. While the Power Law and the Sutherland viscosity models were developed based on the intermolecular force law and attractive force, in which the exponent, $s$, and constants $\left(A_{S}, T_{S}, A_{P}\right)$ are determined from the limiting theoretical cases or the limited ranges of temperatures in experiments. Comparing Eqs. (1) and (4), both of the DSMC and Maxwell viscosity models depend on the molecular mass and diameter leading to the simulation results of the Maxwell viscosity model are close to those of DSMC data while two other viscosity models do not.

\section{CONCLUSIONS}

From the simulation results obtained, whichever the slip and jump boundary conditions are adopted, the viscosity models effect the accuracy of the simulation results of surface gas temperature and slip velocity. The simulation results show that the Maxwell viscosity model provides better predictions of the surface gas temperature and slip velocity than the Sutherland and Power Law viscosity models in comparing with the DSMC data, and pointed out the importance of the viscosity in rarefied gas flow simulations. A good viscosity model will increase the accuracy of the N-S-F simulations for rarefied gas flows, and gives better prediction the peak surface gas temperature to design the thermal protection system in hypersonic vehicles.

\section{ACKNOWLEDGEMENTS}

This research is funded by Vietnam National Foundation for Science and Technology Development (NAFOSTED), under grant number 107.03-2018.27.

\section{REFERENCES}

[1] N. T. P. Le, C. J. Greenshields, and J. M. Reese. Evaluation of nonequilibrium boundary conditions for hypersonic rarefied gas flows. Progress in Flight Physics, 3, (2012), pp. 217-230. https://doi.org/10.1051/eucass/201203217.

[2] G. N. Patterson. Molecular flow of gases. John Wiley and Sons, (1956).

[3] C. R. Lilley and M. N. Macrossan. DSMC calculations of shock structure with various viscosity laws. In The twenty-third Proceeding International Symposium Rarefied Gas Dynamics, (2003), pp. 663-670.

[4] K. B. Jordan. Direct numeric simulation of shock wave structures without the use of artificial viscosity. PhD thesis, Marquette University, (2011).

[5] H. Alsmeyer. Density profiles in argon and nitrogen shock waves measured by the absorption of an electron beam. Journal of Fluid Mechanics, 74, (3), (1976), pp. 497-513. https://doi.org/10.1017/s0022112076001912. 
[6] M. N. Macrossan. $\mu$-DSMC: a general viscosity method for rarefied flow. Journal of Computational Physics, 185, (2), (2003), pp. 612-627. https://doi.org/10.1016/s0021-9991(03)00009-3.

[7] A. M. Mahdavi, N. T. P. Le, E. Roohi, and C. White. Thermal rarefied gas flow investigations through micro-/nano-backward-facing step: Comparison of DSMC and CFD subject to hybrid slip and jump boundary conditions. Numerical Heat Transfer, Part A: Applications, 66, (7), (2014), pp. 733-755. https://doi.org/10.1080/10407782.2014.892349.

[8] A. Mohammadzadeh, E. Roohi, H. Niazmand, and S. K. Stefanov. Detailed investigation of thermal and hydrodynamic flow behaviour in micro/nano cavity using DSMC and NSF equations. In The nineth Proceeding International ASME Conference Nanochannels, Microchannels and Minichannels. American Society of Mechanical Engineers, (2011), pp. 341-350.

[9] J. N. Moss and G. A. Bird. Direct simulation Monte Carlo simulations of hypersonic flows with shock interactions. AIAA Journal, 43, (12), (2005), pp. 2565-2573. https://doi.org/10.2514/1.12532.

[10] A. J. Lofthouse, L. C. Scalabrin, and I. D. Boyd. Velocity slip and temperature jump in hypersonic aerothermodynamics. Journal of Thermophysics and Heat Transfer, 22, (1), (2008), pp. 3849. https://doi.org/10.2514/1.31280.

[11] J. C. Maxwell. On stresses in rarefied gases arising from inequalities of temperature. Philosophical Transactions of the Royal Society, 170, (1878), pp. 231-256.

[12] M. Smoluchowski von Smolan. Üeber wärmeleitung in verdünnten gasen. Annalender Physik und Chemie, 64, (1898), pp. 101-130.

[13] N. T. P. Le and E. Roohi. A new form of the second-order temperature jump boundary condition for the low-speed nanoscale and hypersonic rarefied gas flow simulations. International Journal of Thermal Sciences, 98, (2015), pp. 51-59. https://doi.org/10.1016/j.ijthermalsci.2015.06.017.

[14] OpenFOAM. http://www.openfoam.org. Accessed 12/2018.

[15] E. H. Kennard. Kinetic theory of gases. McGraw-Hill, (1938).

[16] S. Chapman and T. G. Cowling. The mathematical theory of non-uniform gases. Cambridge University Press, (1970).

[17] N. T. P. Le, C. White, J. M. Reese, and R. S. Myong. Langmuir-Maxwell and LangmuirSmoluchowski boundary conditions for thermal gas flow simulations in hypersonic aerodynamics. International Journal of Heat and Mass Transfer, 55, (19-20), (2012), pp. 5032-5043. https://doi.org/10.1016/j.ijheatmasstransfer.2012.04.050. 
